\title{
Acknowledgement of Reviewers for 2020
}

\author{
Petra van Steenbergen ${ }^{1}$
}

Published online: 5 March 2021

(c) The Author(s), under exclusive licence to Springer Nature Switzerland AG part of Springer Nature 2021

International Journal of Geosynthetics and Ground Engineering (IGGE) has several special features and it has maintained a high quality since its establishment in 2015. It is highly appreciated by the researchers who contribute their valuable scientific work to the journal and also by the readers of the journal. Geosynthetics are often used in field projects in combination with some aspects of ground engineering. Because of this special feature, the journal is also read by practising engineers worldwide in addition to researchers, engineering students and other subject-related professionals.

The successful progress of the journal would not have been possible without full support from the reviewers through their sincere contribution in terms of significant time and effort with their valuable technical comments/suggestions to the assessment/review process for the submitted manuscripts. The Editorial Board of IGGE acknowledges this by publishing a list of reviewers. The list below gives the names of all known reviewers of accepted and rejected manuscripts in 2020. We thank you all.

Anubhav
Samer Adeeb
Mohammed Al-Khazaali
Zainab Almheiri
Mohammad Alobaidi
Vivi Anggraini
Jotisankasa Apiniti
Arya Assadi-Langroudi
Dimitrios Atmatzidis
Efthymios Balomenos
Prabir Basudhar
Damian Beben
Christoper Beckett
K. S. Beena
Shobha Bhatia
Ram Rathan Lal Birali
Lalit Borana
Sanandam Bordoloi
Mounir Bouassida
Philippe Bourdeau
Zijun Cao
J. C. Chai
Babloo Chaudhary

Anubhav

Samer Adeeb

Mohammed Al-Khazaali

Zainab Almheiri

Mohammad Alobaidi

Vivi Anggraini

Jotisankasa Apiniti

Arya Assadi-Langroudi

Dimitrios Atmatzidis

Efthymios Balomenos

Prabir Basudhar

Damian Beben

Christoper Beckett

K. S. Beena

Shobha Bhatia

Ram Rathan Lal Birali

Lalit Borana

Sanandam Bordoloi

Mounir Bouassida

J. C. Chai

Babloo Chaudhary

Sowmiya Chawla
Gang Cheng
Zhiliang Cheng
Hariprasad Chennarapu
Liang Cui
Sabatino Cuomo
Utpal Das
Sujit Dash
Manoj Datta
Abhijit Deka
Yongfeng Deng
Francois Duhaime
Rakesh Kumar Dutta
Hany El Naggar
Mohamed Elsawy
Diego Fagundes
Ahmed Fahmy
William Fedrigo
Marek Foglar
Dodagoudar G
Lei Gao
Yanbin Gao
Soheil Ghadr

Kai Gu

Erol Guler

Ashok Gupta

Xiaole Han

Eiji Harada

Kianoosh Hatami

Amarnath Hegde

Abuel-Naga Hossam

Ravi Jakka

Jagadanand Jha

Ni JunJun

Denis Kalumba

Viroon Kamchoom

Rajagopal Karpurapu

Abbas Khayyer

Sreevalsa Kolathayar

Prabir Kolay

Ashutosh Kumar

Jyant Kumar

Vinod Kushvaha

Seyed Hamid Lajevardi

Borana Lalit

Gali Madhavi Latha

Petra van Steenbergen

petra.vansteenbergen@springer.com

1 Springer, Dordrecht, The Netherlands 
Brian Leis

Ben Leshchinsky

Biao Li

Jie Li

Chun Liu

Maria Lopes

Madhira R. Madhav

Priyanka Madhushri

Ioannis Markou

Lindung Zalbuin Mase

Mohamed Meguid

Anil Kumar Mishra

Satyendra Mittal

Yoshihisa Miyata

Nicola Moraci

Ali Morovatdar

Shervin Motamendi

A. Murali Krishna

Kasinathan Muthukkumaran

M. Muthukumar

SeonHong $\mathrm{Na}$

Haslinda Nahazanan

Kok Shien Ng

Sanjay Nimbalkar

Joshua Omer
Ennio Palmeira

Shantanu Patra

Huafu Pei

Ayse Pekrioglu Balkis

Rakesh Pillai

Arun Prasad

Anand Puppala

Reza Rafiee-Dehkharghani

Dhiraj Raj

B. Rao

Krishna Reddy

Narala Gangadhara Reddy

Ganesh Kumar S

Pragyan Sahoo

Narendra Samadhiya

Rajesh Sathiyamoorthy

Vishwas A. Sawant

Ali Shafiee

Mohamed Shahin

Anil Sharma

Mahesh Sharma

Neha Shrivastava

Sanjay Kumar Singh

Nagaratnam Sivakugan

Maciej Sobatka
S. Sreedeep

Pietro Stefanizzi

Christos Stratakos

Peter Sun

Dao-Yuan Tan

Chaosheng Tang

Julian Tao

Fumio Tatsuoka

Vikas Thakur

Jimmy Thomas

Gaurav Tiwari

Ashutosh Trivedi

B. Umashankar

B. V. S. Viswanadham

Yijie Wang

Kuo-Hsin Yang

Erol Yilmaz

Zhen-Yu Yin

Fusheng Zha

Cheng-Cheng Zhang

Yang Zhang

Jun-Jie Zheng

Wan-Huan Zhou

We apologize for any errors or inadvertent omissions

Publisher's Note Springer Nature remains neutral with regard to jurisdictional claims in published maps and institutional affiliations. 